\title{
Transfer function treatment of leptonic tau decays in the Matrix Element method
}

\author{
C. Bélanger-Champagne*, Claus Buszello, Tord Ekelöf \\ Uppsala Universitet \\ E-mail: camilledfnal.gov
}

\begin{abstract}
We report on a preliminary study of the potential of the Matrix Element method to provide a precise determination of the charged Higgs mass if it is produced in decays of the top quark and itself decays to a $\tau$ lepton and a neutrino. This study is done on simulated signal events, including a full simulation of the D0 detector effects. The focus of the work is the development of a transfer function that describes the relationship between the energy of the $\tau$ lepton and its daughter electron. We conclude that it is possible to describe the $\tau$ decay and detector resolution effects using a single transfer function. This study demonstrates that, for a sample containing signal events only, the charged Higgs mass can be determined with better than 7\% accuracy in the mass range $80-140 \mathrm{GeV}$. In order to investigate what charged Higgs mass resolution could be obtained in a future analysis with real data, a study would need to be made that is based on a simulated event sample containing also all relevant backgrounds.
\end{abstract}

Third International Workshop on Prospects for Charged Higgs Discovery at Colliders - CHARGED2010, September 27-30, 2010

Uppsala Sweden

\footnotetext{
* Speaker.
} 


\section{Introduction}

The Matrix Element (ME) method is a powerful multivariate analysis technique that was developed to extract results with maximal statistical significance from small data samples by using all the measured kinematic information contained in these events. It was first used to improve the precision of the top mass measurement at the Tevatron [1]. The characteristics of this method make it a potentially interesting tool in the search for the light charged Higgs boson or in the measurement of its properties, once discovered. It is particularly relevant to consider using this method at the Tevatron, where the available sample of top quark decays will be limited, even after the full run is complete. In this paper we describe a preparatory study performed on simulated events, where the potential of the ME method to provide a measurement of the charged Higgs mass in a specific decay channel was studied. The investigation was done using MadWeight 2.1.11 [2] to calculate the ME weights that constitute the core of the analysis method.

\section{The ME method}

In the Matrix Element method, every event is assigned a weight based on the likelihood that it was produced via the physical process under consideration, given a model with a set of parameters $\alpha$. In this study, $\alpha$ is the mass of the charged Higgs boson. By construction, the ME method can only be applied to exclusive decay channels. The probability weight is driven by the matrix element for the process, giving the method its name. We define $x$ to be the complete set of experimentally measured quantities for a given dataset, and $y$ the corresponding quantities at the partonic level. The probability associated to the matrix element can be expressed as

$$
P(x, \alpha)=N_{\alpha} \int d \phi(y) d z_{1} d z_{2} f\left(z_{1}\right) f\left(z_{2}\right)\left|\mathscr{M}_{\alpha}\right|^{2}(y) T(x, y)
$$

where $N_{\alpha}$ is a factor, typically $\alpha$-dependent, that ensures that $P(x, \alpha)$ is a normalized probability density, $d \phi(y)$ is the multi-dimensional phase-space integration measure, $f\left(z_{1}\right) f\left(z_{2}\right)$ are the parton distribution functions of the two interacting partons, which are also integrated over, $\left|\mathscr{M}_{\alpha}\right|^{2}(y)$ is the squared matrix element amplitude and $T(x, y)$ is the resolution or transfer function that relates the experimentally measured quantities to the partonic quantities. The value of the model parameters $\alpha$ are then measured by maximizing the likelihood $\mathscr{L}(\alpha)$ constructed from the combined event probabilities. In practice, for a sample of $N$ events, one minimizes the negative natural logarithm of $\mathscr{L}(\alpha)$, given by:

$$
-\ln \mathscr{L}(\alpha)=-\sum_{i=1}^{N} \ln P\left(x_{i}, \alpha\right)+N \cdot R(\alpha)
$$

where terms that do not depend on $\alpha$ are omitted. The $R(\alpha)$ term incorporates in the likelihood all detector acceptance effects. The value of $R(\alpha)$ can be estimated by

$$
R(\alpha)=\frac{N_{\text {selected }}(\alpha)}{N_{\text {generated }}(\alpha)}
$$

where $N_{\text {generated }}(\alpha)$ is the number of events generated in a Monte Carlo event sample with model parameters $\alpha$ and $N_{\text {selected }}(\alpha)$ is the number of those events that pass the event selection used for the analysis. A complete and detailed description of the method can be found in References $[3,4,5]$. 


\section{Light charged Higgs mass measurement}

We study the process in which a light charged Higgs is produced in the decay of one of the top quarks in a $t \bar{t}$ pair. The charged Higgs decays to a $\tau$ lepton and a neutrino and the $\tau$ lepton decays in turn to an electron and two more neutrinos. The other top quark decays to a $W$ boson and a bottom quark, with the $W$ decaying hadronically. The complete decay chain is illustrated in Figure 1.

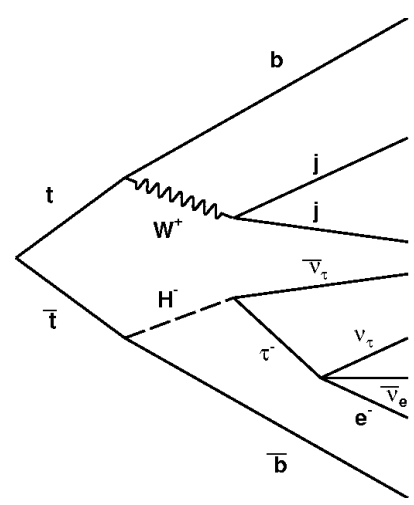

Figure 1: Diagram of the process of light charged Higgs production in $t \bar{t}$ decays with $\tau$ decay to a final-state electron.

This study is performed using simulated events for the signal process only. They are generated with Pythia [6, 7]. The samples were generated with charged Higgs masses of 80, 100, 120 and $140 \mathrm{GeV}$, with approximately 200000 events generated for each mass. The generated events ran through a simulation of the D0 detector [8] response and were reconstructed using the standard D0 algorithms.

A cut-based selection is applied to the events, designed to enhance the signal and match the topology that the matrix element describes. The events are required to have

- Exactly one isolated electron with $p_{T}>20 \mathrm{GeV}$,

- Exactly 4 jets with transverse energy $E_{T}>20 \mathrm{GeV}$,

- At least $20 \mathrm{GeV}$ of missing transverse energy.

For this study based on simulated events, we also require that the four jets and the electron be matched to partonic equivalents in the event generator record. The selection efficiency gives an estimate of the acceptance factor $R(\alpha)$. It varies between $0.5 \%$ and $0.7 \%$ in the $m_{H^{ \pm}}$range studied. We extrapolated between the mass points where samples were generated to produce an acceptance curve that varies smoothly with $\alpha$ to be used in the likelihood calculation of Equation (2.2).

\section{Mass determination from partonic to fully simulated events}

The first test of the capacity of the ME method to provide a mass measurement in the channel of interest is to compute the ME likelihood using simulated events at partonic level. The partonic 
4-momenta of the particles are used as input data to the method and the $\tau$ is treated as stable so its 4-momentum can be used directly.

To compute the ME likelihood using fully simulated events, transfer functions need to be included for jets. MadWeight allows the inclusion of transfer functions in energy, pseudorapidity $\eta$ and azimuthal angle $\phi$. However, the spatial components $\eta$ and $\phi$ are measured accurately enough in the detector so the only significant component of the transfer function is that describing the relationship between partonic and reconstructed energies.

Jet transfer functions are measured separately for light jets and b-jets using both the signal samples and a Standard Model top pair production sample. The difference between the energy of the quark that is associated to the jet and the reconstructed jet energy is fitted, in bins of reconstructed jet energy and pseudorapidity, with a double Gaussian. Within each pseudorapidity region, the dependence of the parameters of the double Gaussian on the reconstructed jet energy is fitted in turn with a straight line to produce a smoothly varying transfer function. Such a parametrization has been done before at D0 [9]. An example of a double Gaussian fit of the quark-jet energy difference is shown in Figure 2a. The transfer functions parameters derived from the two samples are compatible. The behavior of these transfer functions is tested by computing the ME likelihood on hybrid events where the 4-momentum of the partonic $\tau$ is used but the 4-momenta of the jets are taken at the reconstructed level.

The computational complexity of the ME method is a limiting factor in its application to processes with many final state particles. Both the derivation of the ME and the integration over many parameters of the final state can become problematic. In order to reduce this complexity we use the $2 \rightarrow 6 \mathrm{ME}$ with the $\tau$ lepton treated as stable in the final state instead of the $2 \rightarrow 8 \mathrm{ME}$ that would include the $\tau$ decay to an electron and two neutrinos. To account for this decay, we use a transfer function that associates the electron, measured in the detector simulation, to the $\tau$ at parton level. This is atypical, as transfer functions are generally chosen to only parametrize detector effects. In this transfer function we parametrize the behavior of neutrinos that are aligned with their mother particle. We observe that the reconstructed position of the electron in the detector matches accurately that of the $\tau$, so we need only to use the energy component of the transfer function. This transfer function parametrizing neutrinos is of interest beyond our specific study as the method can be applied to leptonic $\tau$ decays in other processes as well as other decays that feature aligned neutrinos. We observe the energy difference distribution separately for events with $e^{+}$and $e^{-}$in the charged Higgs samples and observe that the distribution is well described by a Moyal distribution [10]. An example of an electron-to- $\tau$ transfer function is shown in Figure $2 b$. We find that the fit parameters obtained for all samples and for events with $e^{+}$and $e^{-}$are compatible. We test the behavior of this transfer function by computing the ME likelihood using hybrid events where the 4-momentum of the reconstructed electron and its associated transfer function is used while the quark 4-momenta are used for the "jets".

Finally, the ME likelihood is calculated with both types of transfer functions, using fully simulated and reconstructed jets and electron, to complete this study.

\section{Results and Outlook}

The ME likelihood was calculated for all four simulated charged Higgs masses with all four 


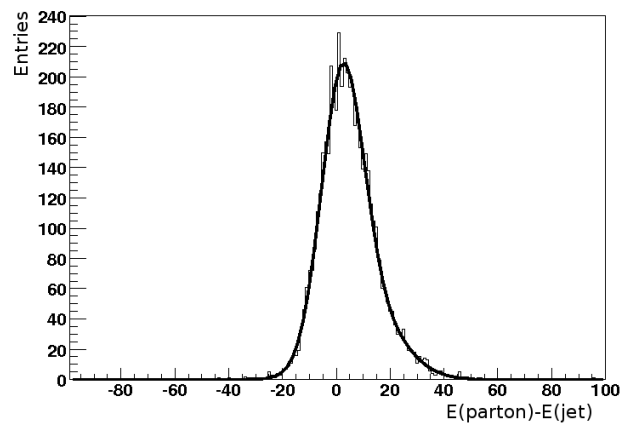

(a)

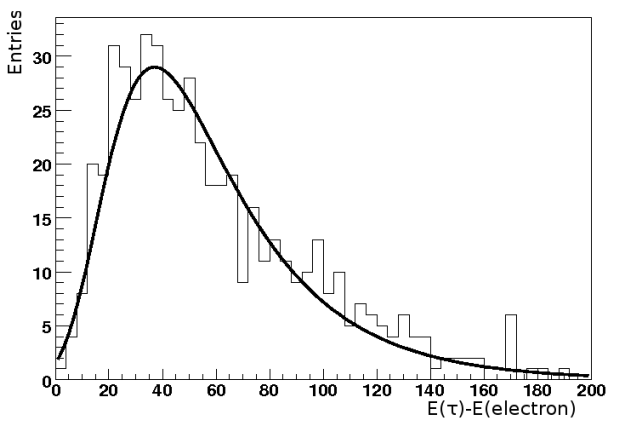

(b)

Figure 2: (a) Example transfer function fit for jets, here light quark jets in the energy range 40$50 \mathrm{GeV}$. (b) Transfer function fit for the electron-to- $\tau$ transfer function.

types of events: partonic, two hybrid event configurations and fully simulated. The mass range around the minimum of the negative logarithm of the likelihood was fitted with a second-order polynomial to determine the most likely charged Higgs mass. Example likelihood fits for the four event types using the event sample with a charged Higgs mass of $80 \mathrm{GeV}$ are shown in Figure 3. The full set of the resulting fitted most likely masses is given in Table 1 .

Table 1: Most likely mass according to ME method for various types of simulated events.

\begin{tabular}{|c|cccc|}
\hline $\begin{array}{c}\text { Simulated } \\
\text { mass }(\mathrm{GeV})\end{array}$ & $\begin{array}{c}\text { Partonic events } \\
(\mathrm{GeV})\end{array}$ & $\begin{array}{c}\text { Fully simulated } \\
\text { jets only }(\mathrm{GeV})\end{array}$ & $\begin{array}{c}\text { Fully simulated } \\
\tau \text { decay only }(\mathrm{GeV})\end{array}$ & $\begin{array}{c}\text { Fully simulated } \\
\text { events }(\mathrm{GeV})\end{array}$ \\
\hline 80 & 84.5 & 84.6 & 78.1 & 80.4 \\
100 & 101.7 & 104.2 & 97.4 & 100.8 \\
120 & 120.5 & 121.5 & 117.7 & 114.4 \\
140 & 139.7 & 136.0 & 137.8 & 130.2 \\
\hline
\end{tabular}

With partonic events, we see a good match between the simulated charged Higgs mass and the most likely mass found with the ME method at all tested masses. However, there is a systematic, mass-dependent shift that is inherent to the method. The largest difference occurs for the case where the charged Higgs is taken to be $80 \mathrm{GeV}$, very close to the mass of the $W$ boson. On hybrid events for which fully simulated jets are used, and a jet transfer function is applied to account for detector resolution, we observe that the added effect on the determination of the most likely mass via the ME method is comparatively small. For the other type of hybrid events, where the fully simulated $\tau$-toelectron decay is treated with a transfer function that describes both the detector resolution and the relationship between the energy of the $\tau$ and its daughter electron, we observe that the most likely mass value is pulled down, but remains close to the generated mass. In comparison to the jet transfer functions, which are obtained using a well-established method with many detailed adjustments such as energy-dependence and division according to detector geometry, the electron-to- $\tau$ transfer function is new to this analysis. It did not include the dependence of the function parameters on electron energies or on detector regions. It is possible to include such refinements to the electron- 


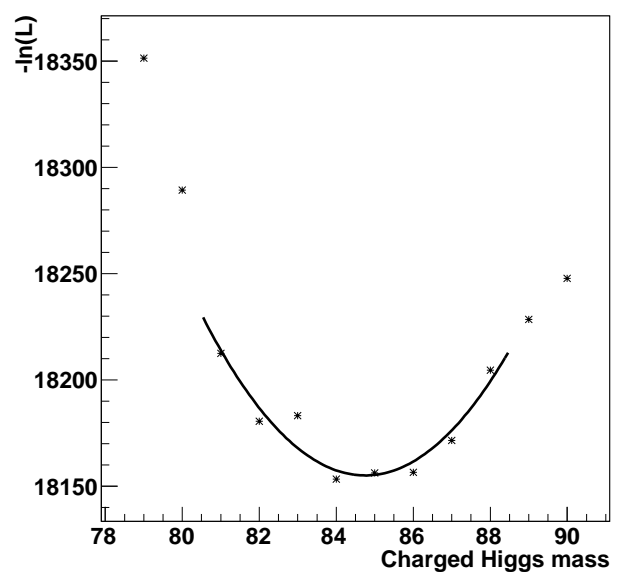

(a)

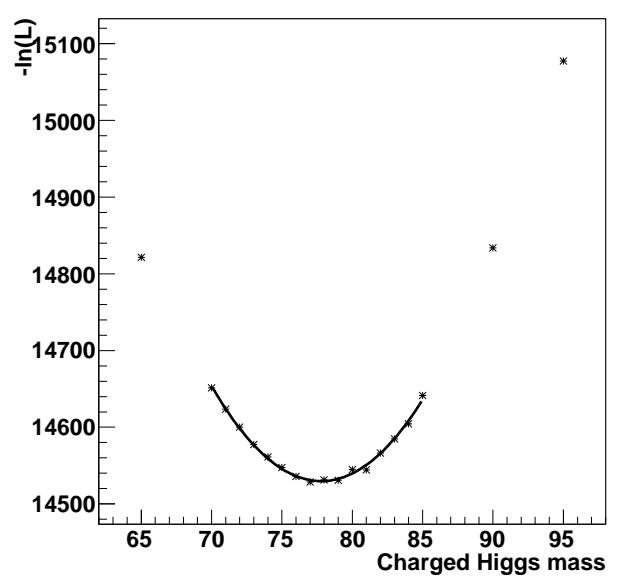

(c)

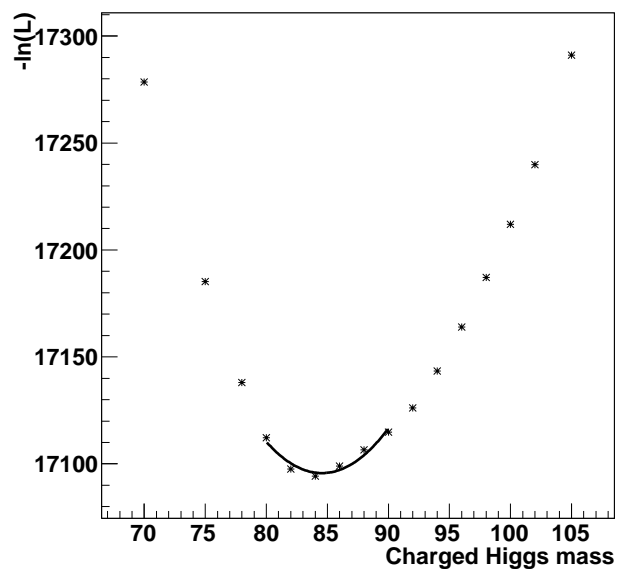

(b)

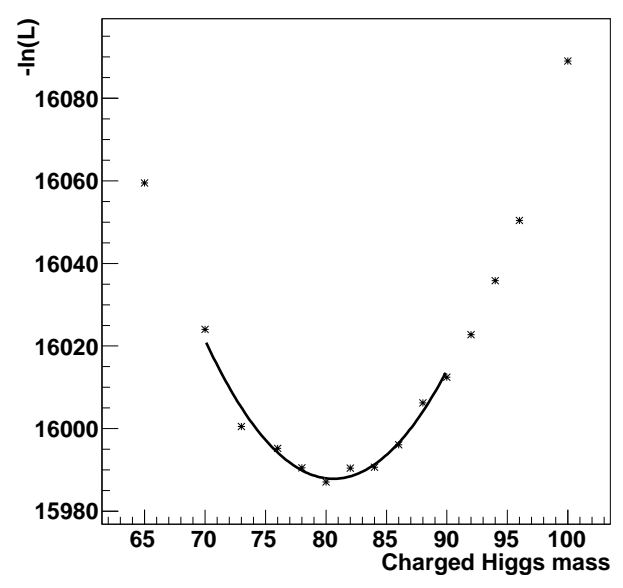

(d)

Figure 3: Likelihood fits for events with $m_{H^{ \pm}}=80 \mathrm{GeV}$ (a) at parton level, (b) with simulated jets only, (c) with simulated $\tau$ decays to electron only and (d) at fully simulated level.

to- $\tau$ transfer function in the future. They could provide improved accuracy and resolution. Finally, complete detector events were used with transfer functions applied both to jets and to the electron. The difference between the measurement of the most likely charged Higgs mass and the simulated charged Higgs mass varies between 0 and $10 \mathrm{GeV}$ in the charged Higgs mass range 80-140 GeV. All mass determinations for these signal event samples are accurate to better than $7 \%$ of the generated charged Higgs mass. The observed bias has been found to be present at a comparable level in earlier studies of other physics processes and seems to be inherent to the method. A study like the present one can be used to account and correct for this bias in a measurement performed on data by extracting a correction factor based on the raw measured mass. Our study demonstrates that it is possible to use a transfer function to model decays of $\tau$ leptons to electrons in the ME 
method. This extends the use of the transfer function beyond the traditional task of modeling the detector resolution. This study is intended as a first step in the process of demonstrating that the ME method can be used to provide a measurement of the charged Higgs mass when produced in decays of the top quark. Further studies are needed to make a complete assessment. In particular, inclusion of background events is necessary to determine the mass resolution achievable with the ME method for the light charged Higgs mass measurement. There is also room for improvement in the electron-to- $\tau$ transfer function: the variation of the function parameters with electron energy and between different pseudorapidity regions in the detector could be studied and included in the transfer function.

\section{Acknowledgments}

The speaker would like to thank the D0 Collaboration for the permission to use simulated charged Higgs bosons events that include a full simulation of the D0 detector.

\section{References}

[1] V. M. Abazov et al. [D0 Collaboration], A precision measurement of the mass of the top quark, Nature 429, 638 (2004) [hep-ex/ 0406031$].$

[2] MadWeight webpage, http://cp3wks05.fynu.ucl.ac.be/twiki/bin/view/Software/MadWeight

[3] J. C. Estrada Vigil, Maximal use of kinematic information for the extraction of the mass of the top quark in single-lepton t anti-t events at D0, FERMILAB-THESIS-2001-07 (2001).

[4] M. F. Canelli, Helicity of the $W$ boson in single-lepton $t \bar{t}$ events, FERMILAB-THESIS-2003-22 (2003).

[5] C. Garcia, Precision Measurement of the Mass of the Top Quark in p $\bar{p}$ Collisions, FERMILAB-THESIS-2007-21 (2007).

[6] T. Sjostrand, S. Mrenna and P. Z. Skands, PYTHIA 6.4 Physics and Manual, JHEP 05, 026 (2006) [hep-ph/0603175].

[7] J. Alwall, C. Biscarat, S. Moretti, J. Rathsman and A. Sopczak, The $p \bar{p} \rightarrow t b H^{ \pm}$process at the Tevatron in HERWIG and PYTHIA, Eur. Phys. J. C 39S1, 37 (2005) [hep-ph/0312301].

[8] V. M. Abazov et al. [D0 Collaboration], The Upgraded Do Detector, Nucl. Instrum. Meth. A565, 463-537 (2006) [physics/0507191]

[9] V. M. Abazov et al. [D0 Collaboration], Measurement of the top quark mass in the lepton+jets channel using the matrix element method on $3.6 \mathrm{fb}^{-1}$ of DO Run II data, D0 Conference Note 5877-CONF

[10] J. E. Moyal, Theory of Ionization Fluctuations, Phil. Mag. 46, 263 (1955) 